\title{
Presencia de plaguicidas organoclorados en forraje para ganado en unidades de producción de leche orgánica en Tecpatán, Chiapas
}

\section{Presence of organochlorine pesticides in forage for cattle in organic milk production units in Tecpatan, Chiapas}

\author{
María N. Murga J uáreza , Rey Gutiérrez Tolentino ${ }^{b *}$, Salvador Vega y León ${ }^{b}$, J osé J. Pérez González ${ }^{b}$, Beatriz \\ Schettino Bermúdez ${ }^{b}$, J orge L. Ruíz Rojasc ${ }^{c}$ Alberto Yamazaki Mazac
}

\section{RESUMEN}

La presencia de plaguicidas organoclorados en el ambiente es preocupante por su persistencia, potencial de transporte a larga distancia y grado de toxicidad para la fauna y los seres humanos. En México, Chiapas ocupa el primer lugar en la producción de alimentos orgánicos, y Tecpatán es el principal municipio productor de leche orgánica. El objetivo de este trabajo fue determinar la presencia de plaguicidas organoclorados en forraje para ganado lechero en unidades de producción de leche orgánica. Se eligieron tres unidades de producción de leche orgánica y se colectaron muestras de forraje y leche en cada unidad. Los plaguicidas se extrajeron y purificaron con base en lo establecido por la USEPA (United States Environmental Protection Agency) y se analizaron por cromatografía de gases con detector de captura de electrones. Los compuestos que rebasaron el límite máximo permisible según el Codex Alimentarius fueron lindano, heptacloro + epóxido de heptacloro y aldrín + dieldrín (37.5 $\pm 84,62 \pm 125$ y $20 \pm 50$ ng.g ${ }^{-1}$ base grasa, respectivamente). En las muestras de forraje los valores más altos fueron alfa + beta-HCH, lindano y heptacloro + epóxido de heptacloro $\left(76.3 \pm 140,43 \pm 105 \mathrm{ng} \cdot \mathrm{g}^{-1}\right.$ y $40 \pm 76 \mathrm{ng} \cdot \mathrm{g}^{-1}$ peso seco). Es probable que la destacada presencia de estos compuestos se deba a su utilización en el control de vectores en campañas de salud o de ectoparásitos del ganado de la región por parte de productores convencionales.

PALABRAS CLAVE: Plaguicidas organoclorados, Forraje, Producción orgánica, Chiapas.

\section{ABSTRACT}

The presence of organochlorine pesticides in the environment is a current topic due to the persistence, potential for long-range transport and toxicity to wildlife and humans. In Mexico, Chiapas has the first place in the production of organic food, where Tecpatan municipality is the first place of organic milk. Twenty-five years ago the farmers did not use organochlorine pesticides, but some conventional farmers employ this compounds for controlling pests and disease vectors with knowledge of the health authorities. The aim of this study was to determine the presence of organochlorine pesticides in feed for dairy cattle production of organic milk. Three production units of organic milk samples were chosen to collect fodder and milk for one year, considering wet and dry season. Pesticides were extracted and purified based on the provisions of the USEPA (United States Environmental Protection Agency) and analyzed by gas chromatography with electron capture detector (ECD $\left.{ }^{63} \mathrm{Ni}\right)$. The compounds that exceeded the maximum permissible limit by the Codex Alimentarius were lindane, heptachlor and heptachlor epoxide + aldrin + dieldrin ( 37.5 $\pm 84,125 \pm 62$ and $20 \pm 50$ basis $\mathrm{ng} \cdot \mathrm{g}^{-1}$ fatty base, respectively). In the forage samples the higher values were alpha + beta-HCH,

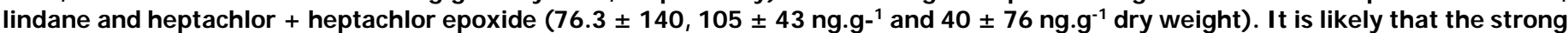
presence of these compounds is due to their use in vector control in health campaigns or ectoparasites of livestock in the region by conventional producers.

KEY WORDS: Organochlorine pesticides, Forage, Organic production, Chiapas.

Recibido el 1 de abril de 2015. Aceptado el 19 de mayo de 2015

Ciencias Agropecuarias, Universidad Autónoma Metropolitana, Xochimilco. México.

Departamento de Producción Agrícola y Animal. Universidad Autónoma Metropolitana, Xochimilco. Calzada del hueso, 1100, Col. Villa Quietud. Delegación Coyoacán 04960. CDMX, México. Tel. 54837000 Ext. 3054

Facultad de Medicina Veterinaria y Zootecnia, Universidad Autónoma de Chiapas. México.

Autor de correspondencia: reygut@correo.xoc.uam.mx. 


\section{INTRODUCCIÓN}

Los plaguicidas organoclorados (PO) son compuestos químicos que presentan alta estabilidad física y química, solubilidades variables en agua, volátiles, altamente solubles en disolventes orgánicos y afines a los tejidos o compuestos grasos de los alimentos. Estas características favorecen su persistencia en el ambiente $y$ su lenta biodegradabilidad ${ }^{(1)}$. Al momento de ser emitidos en el ambiente, los PO son volatilizados hacia la atmósfera y en función de las condiciones del viento, la humedad y la temperatura, se pueden desplazar a zonas más alejadas. Se depositan en forma húmeda (lluvia) o seca (polvo) en la vegetación, el suelo y el agua más próximos ${ }^{(2,3)}$. La contaminación en la vegetación sucede de dos maneras: a) por deposición atmosférica y la interacción de las ceras y las grasas propias de las plantas en las hojas; o b) por su absorción en la raíz y su transporte a la parte superior de la planta. Existen ciertas características de las plantas como la pilosidad y el contenido de cera de la cutícula, que determinan el grado de acumulación de partículas lipofílicas asociadas con los contaminantes orgánicos ${ }^{(4)}$.

La exposición a PO se da por tres vías, respiración, absorción dérmica y consumo de alimentos contaminados, siendo esta última la de mayor impacto. Los rumiantes lecheros están expuestos por el consumo de forraje y suelo contaminados. Pequeñas cantidades de PO entran en el cuerpo de los rumiantes, se concentran en el tejido graso y se transportan a través de las grasas y lipoproteínas circulantes del organismo para posteriormente ser excretados por orina y leche $e^{(5,6,7)}$.

En la actualidad las preferencias de los consumidores han cambiado en la búsqueda de alimentos más sanos y de un sistema de producción amigable con el ambiente. Ante esta perspectiva, se encuentran la agricultura y la ganadería orgánica, donde se busca minimizar el uso de insumos externos de síntesis química, promover el incremento de la fertilidad del suelo, la conservación del agua, la biodiversidad y la salud de los consumidores ${ }^{(8,9)}$.
Los alimentos orgánicos son aceptados rápidamente en el mercado mundial de alimentos (hortalizas, frutas, miel y leche entre otros). México se encuentra entre los países que han experimentado un incremento superior al $25 \%$ en la superficie que se cultiva de manera orgánica, tanto en agricultura como en ganadería. En este sentido, Chiapas ocupa el primer lugar nacional en la producción de alimentos orgánicos, entre los cuales destacan café, miel, cacao y leche. El municipio de Tecpatán es el más importante tanto a nivel estatal como nacional, en la producción de leche orgánica, la cual se produce bajo un sistema de producción donde los animales son criados bajo prácticas que no contemplan el uso de agroquímicos sintéticos (fertilizantes, plaguicidas, hormonas, transgénicos, entre otros) ${ }^{(10)}$. De esta manera, se pretende obtener un producto que, entre otras características sea inocuo, es decir libre de residuos y contaminantes químicos ${ }^{(11)}$. Sin embargo, la persistencia de contaminantes en el ambiente como los PO, pueden alterar esta inocuidad; por tal motivo el objetivo del presente trabajo fue conocer los niveles de plaguicidas organoclorados en muestras de forraje para consumo de ganado lechero, en unidades de producción de leche orgánica en el municipio de Tecpatán, Chiapas.

\section{MATERIAL Y MÉTODOS}

El municipio de Tecpatán, se localiza al noreste del estado de Chiapas a $17^{\circ} 23^{\prime} 09^{\prime \prime}$ N y $93^{\circ} 52^{\prime} 35^{\prime \prime} \mathrm{O}$ y altitud de $300 \mathrm{msnm}$. Clima cálido húmedo con temperatura mínima promedio anual de $18{ }^{\circ} \mathrm{C}$, máxima promedio anual de $34{ }^{\circ} \mathrm{C}$ y media anual de $26{ }^{\circ} \mathrm{C}$. Lluvias todo el año en la zona norte del municipio con una precipitación pluvial promedio anual de 1,885 mm. Los vientos van de norte a sur y viceversa dependiendo de la estación del año, y un relieve accidentado ${ }^{(12)}$.

Se seleccionaron al azar tres unidades de producción de leche orgánica (R1, R2 y R3) de un total de 30 productores. En cada unidad, se tomaron 18 muestras de forraje. Para el caso de la leche, además de las tres unidades de producción, se tomó una muestra del tanque colector (R4), dando un total de 24 muestras. Dichas muestras se tomaron 
de abril de 2012 a febrero de 2013, cubriendo así las épocas de seca (abril, diciembre y febrero) y lluvia (junio, agosto y octubre).

De acuerdo a información recabada mediante encuestas realizadas en la zona de estudio, la raza de las vacas de las unidades de producción fue Cebú-Suizo en R1 y R2, y Holstein-Cebú en R3; la dieta estaba basada exclusivamente en libre pastoreo todo el día; en la época seca no se proporcionó ningún tipo de suplementación. Los tipos de forraje predominantes en la zona de estudio y por tanto de mayor consumo por parte de los rumiantes, son insurgente (Brachiaria brizantha), mulato (Brachiaria hibrido), señal o chontalpo (Brachiaria decumbens), estrella (Cynodon plectostachium), y cabezón (Eleusine indica). Por otro lado, los suelos predominantes son luvisol, (con un profundo horizonte superficial enriquecido con arcillas), cambisol (con una capa superficial oscura de $25 \mathrm{~cm}$ de espesor y buen contenido de materia orgánica) y litosol (el cual tiene menos de $10 \mathrm{~cm}$ de espesor, color variable, alto contenido de materia orgánica y fertilidad de media a alta). El agua que abastece a las tres unidades de producción proviene de la Presa Nezahualcóyotl(12).

La superficie utilizada en cada unidad de producción para pastoreo es de $28(\mathrm{R} 1), 19(\mathrm{R} 2)$ y 50 (R3) hectáreas. El número de vacas con el que se cuenta es de 42, 20 y 36 en R1, R2 y R3 respectivamente; utilizadas para doble propósito (leche y carne), desparasitadas cada seis meses con albendazol y febendazol según la prescripción del médico veterinario. Al momento de realizar la toma de muestras ninguna vaca presentó mastitis, sin embargo, cuando existe un problema de este tipo las vacas son tratadas con antibióticos y retiradas por 40 días $^{(8)}$.

\section{Toma de muestras}

Forraje. En cada unidad se establecieron 15 puntos de muestreo con base en la Norma Oficial Mexicana NMX-Y-111-SCFI-2010(13). Las muestras se mezclaron para tomar una muestra compuesta final de aproximadamente $1 \mathrm{~kg}$.

Leche. La toma de muestras de leche se realizó en cuatro unidades de producción ( $R 1, R 2$, R3 y R4) con base en lo establecido por la Norma Oficial Mexicana NMX-F-718-COFOCALEC-2006(14). Se colectó una muestra de la primera ordeña, en un volumen de $500 \mathrm{ml}$.

Ambas muestras, (forraje y leche) se transportaron en hielera a una temperatura de $4{ }^{\circ} \mathrm{C}$ hasta el Laboratorio de Análisis Instrumental de la Universidad Autónoma Metropolitana unidad Xochimilco, donde fueron procesadas y analizadas. Las muestras de forraje se secaron en un horno a 40 ${ }^{\circ} \mathrm{C}$, se molieron en un macro molino y se tamizaron en una malla de $1 \mathrm{~mm}$. Para la extracción de los compuestos clorados, se pesaron $5 \mathrm{~g}$ de muestra y se colocaron en un sistema de extracción soxhlet (A50270 Scorpion Scientific) empleando una solución hexano:acetona $(1: 1 \quad \mathrm{v} / \mathrm{v})$ grado cromatográfico (JT Baker) durante $8 \mathrm{~h}$. Al término, el extracto se concentró en un rotovaporador (Buchi Water bath B 480 y rotovapor R-114) a un volumen de $5 \mathrm{ml}$. En las muestras de leche se extrajo la grasa de acuerdo con Frank et a $k^{15)}$ con solución detergente en un baño térmico a $90{ }^{\circ} \mathrm{C}$, conservándola a $-20{ }^{\circ} \mathrm{C}$ para su análisis cromatográfico. La purificación de $\mathrm{PO}$ en las muestras de forraje y leche orgánica se realizó en columnas cromatográficas de vidrio empacadas con fibra de vidrio, florisil ( $13 \mathrm{~g}$ ) y sulfato de sodio ( $5 \mathrm{~g}$ ) y se eluyeron con $20 \mathrm{ml}$ de una mezcla hexano:éter $(8: 2 \mathrm{v} / \mathrm{v})^{(16)}$.

El análisis y la cuantificación se realizó en un cromatógrafo de gases (Shimadzu CG-2010 Plus) equipado con detector de captura de electrones, columna capilar HP-5 de 30 m de longitud, $0.25 \mathrm{~mm}$ de diámetro interno, $0.25 \mu \mathrm{m}$ de grosor de película $5 \%$ fenil metilsilicona; utilizando como gas acarreador helio de alta pureza a un flujo de 0.65 $\mathrm{ml} / \mathrm{min}$ a través de la columna. Las temperaturas del inyector y el detector fueron de $260{ }^{\circ} \mathrm{C}$ y $320^{\circ} \mathrm{C}$, respectivamente. Las rampas de temperatura en el horno fueron de $90{ }^{\circ} \mathrm{C}$ al inicio con una duración de 2 min, seguido de una rampa de $2^{\circ} \mathrm{C} / \mathrm{min}$ llegando a una temperatura de $160{ }^{\circ} \mathrm{C}$ por $3 \mathrm{~min}$, seguido de una rampa de $15{ }^{\circ} \mathrm{C} / \mathrm{min}$ para llegar a $220{ }^{\circ} \mathrm{C}$ durante $3 \mathrm{~min}$, seguido de una rampa de $280^{\circ} \mathrm{C} / \mathrm{min}$ por 2 min para llegar a una temperatura final de 310 ${ }^{\circ} \mathrm{C}$. El volumen de inyección fue de $0.8 \mu \mathrm{l}$ en las muestras leche y $2 \mu$ en forraje en modo "Splitless" 
con un tiempo de corrida de 50 min. El control de calidad se realizó a través de duplicados de las muestras, dos blancos fortificados por cada lote de seis muestras y corridas de blancos electrónicos. Para la identificación de cada compuesto se utilizó un estándar externo con una mezcla de 16 compuestos: alfa, beta, gama y delta-HCH, heptacloro, epóxido de heptacloro, aldrín, dieldrín, endrín, endosulfán I, endosulfán II, DDE (1,1dicloro-2,2-bis (4 clorofenil) etileno), endrín aldehído, DDT (1,1,1-tricloro-2,2-bis (4 clorofenil) etano), DDD (1-cloro-4-(2,2-dicloro-1-(4-clorofenil) etil1) benceno) y endosulfato (Chem Service Inc, West Chester, PA, USA). En el Cuadro 1 se muestra el límite de detección y el porcentaje recobrado para cada plaguicida organoclorado en las muestras de forraje y leche.

El estudio fue de carácter descriptivo, longitudinal. Se realizó un análisis exploratorio de

Cuadro 1. Valores de recobrado y límite de detección en plaguicidas organoclorados

\begin{tabular}{lcc}
\hline Plaguicida & $\begin{array}{c}\text { Límite de } \\
\text { detección } \\
\left(\mathrm{ng} \cdot \mathrm{g}^{-1}\right)\end{array}$ & $\begin{array}{c}\text { Recobrado } \\
(\%)\end{array}$ \\
\hline Alfa-HCH & 0.08 & $76 \pm 6^{*}$ \\
Beta-HCH & 0.16 & $87 \pm 3$ \\
Gama-HCH & 0.08 & $83 \pm 5$ \\
Delta-HCH & 0.08 & $85 \pm 4$ \\
Heptacloro & 0.15 & $75 \pm 8$ \\
Aldrín & 0.08 & $78 \pm 7$ \\
Epóxido de heptacloro & 0.08 & $76 \pm 3$ \\
Endosulfán I & 0.02 & $100 \pm 1$ \\
DDE & 0.08 & $80 \pm 7$ \\
Dieldrín & 0.15 & $78 \pm 4$ \\
Endrín & 0.10 & $87 \pm 6$ \\
Endosulfán II & 0.02 & $105 \pm 1$ \\
DDD & 0.08 & $79 \pm 5$ \\
Endrín aldehído & 0.07 & $85 \pm 6$ \\
Endosulfato & 0.02 & $130 \pm 1$ \\
DDT & 0.08 & $87 \pm 5$ \\
\hline
\end{tabular}

*Promedio y desviación estándar; $\mathrm{HCH}=$ Hexaclorociclohexano, $\mathrm{DDE}=1,1$ dicloro-2,2-bis (4 clorofenil) etileno, DDT=1,1,1-tricloro-2,2-bis (4 clorofenil). etano, $\mathrm{DDD}=$ diclorodifenildicloroetano. datos (frecuencias, valores mínimos y máximos, el valor de promedios, desviaciones estándares) usando SPSS versión 20.0 para Windows.

\section{RESULTADOS Y DISCUSIÓN}

Se encontraron niveles de PO en todas las muestras de forraje y leche orgánica analizadas. De los 16 PO analizados se identificaron 11 (alfa, beta$\mathrm{HCH}$, lindano, heptacloro, aldrin, epóxido de heptacloro, endosulfanes I y II, endosulfato y DDT) en ambas matrices. En el Cuadro 2 se muestra la concentración promedio de los PO identificados en las muestras de leche orgánica. Los compuestos con los valores más altos fueron lindano $37.5 \mathrm{ng} \cdot \mathrm{g}^{-1}(\mathrm{R} 1)$, heptacloro + epóxido de heptacloro $62 \mathrm{ng}^{-\mathrm{g}^{-1}}$ (R2) y aldrín + dieldrín 20 ng. $\mathrm{g}^{-1}$ (R1). Estos valores estuvieron por arriba del límite máximo permisible establecido por el Codex Alimentarius. Los compuestos que se presentaron con mayor frecuencia durante el muestreo fueron heptacloro + epóxido de heptacloro $50 \%$ en todas las unidades de producción, endosulfanes I y II y aldrín + dieldrín $33 \%$. Estos resultados son similares a los encontrados por Hernández et $a{ }^{(17)}$, quienes determinaron la presencia de PO en muestras de leche de bovinos suplementados con residuos de algodón, encontrando frecuencias de $52.6 \%$ (DDT), 38.2 \% (endrín) y $28 \%$ (endosulfán I). Por otro lado, los valores encontrados en este estudio, sobrepasan lo reportado por otros autores $^{(5,7,18)}$ en estudios realizados a nivel nacional e internacional; a excepción del reporte de Díaz et $a^{\left({ }^{(6)}\right.}$ en Colombia, quienes encontraron niveles de 80.1 ng. $\mathrm{g}^{-1}$ de aldrín + dieldrin. Mientras que en el presente estudio la concentración de estos compuestos fue de $5.1 \mathrm{ng} \cdot \mathrm{g}^{-1}$. En general, los valores encontrados no rebasan los límites máximos permisibles establecidos por organismos nacionales e internacionales (Cuadro 3). Estas diferencias probablemente se deban al actual uso de endosulfán en cultivo de jitomate en zonas aledañas a la zona de estudio ${ }^{(19)}$. Aunado a esto, de acuerdo con datos del Ministerio de Salud Pública de Guatemala, en los años 2010 y 2012, hubo una elevación en morbilidad por dengue y malaria $(16,738$ y 27, 755 casos a nivel nacional, respectivamente), y en los años siguientes estas cifras disminuyeron de manera 
Cuadro 2. Presencia de plaguicidas organoclorados en muestras de leche orgánica en cada unidad de producción analizada

\begin{tabular}{|c|c|c|c|c|c|c|}
\hline Compuestos & Unidad & $\begin{array}{l}\text { Promedio } \\
\text { (ng.g-1 base } \\
\text { grasa) }\end{array}$ & $\mathrm{DE}$ & $\begin{array}{c}\text { Intervalo } \\
(\text { ng.g-1) }\end{array}$ & $\begin{array}{c}\text { Frecuencia } \\
\qquad \%)^{a}\end{array}$ & $\begin{array}{c}\text { LMP } \\
\left(\mathrm{ng} \cdot \mathrm{g}^{-1}\right)^{\mathrm{b}}\end{array}$ \\
\hline \multirow{4}{*}{ alfa + beta- $\mathrm{HCH}$} & R1 & 1.6 & 4.0 & $0-9.5$ & 17 & \multirow{4}{*}{$\mathrm{NE}^{\mathrm{d}}$} \\
\hline & R2 & $N D^{b}$ & ND & ND & ND & \\
\hline & R3 & ND & ND & ND & ND & \\
\hline & R4 & 0.79 & 1.9 & $0-4.6$ & 33 & \\
\hline \multirow{4}{*}{ Lindano } & $\mathrm{R} 1$ & 37.5 & 84 & $0-187.0$ & 17 & \multirow{4}{*}{10} \\
\hline & R2 & ND & ND & ND & ND & \\
\hline & R3 & ND & ND & ND & ND & \\
\hline & $\mathrm{R} 4$ & 0.80 & 1.70 & $0-3.98$ & 17 & \\
\hline \multirow{4}{*}{ Heptacloro + epóxido de heptacloro } & R1 & 9.6 & 11.2 & $0-25.5$ & 50 & \multirow{4}{*}{6} \\
\hline & R2 & 62 & 86.3 & $0-221$ & 50 & \\
\hline & R3 & 11 & 20 & $0-45.9$ & 50 & \\
\hline & R4 & 15.5 & 27 & $0-65.6$ & 50 & \\
\hline \multirow{4}{*}{ Aldrin + dieldrín } & $\mathrm{R} 1$ & 21 & 52 & $0-123$ & 33 & \multirow{4}{*}{6} \\
\hline & R2 & 0.31 & 0.49 & $0-1.08$ & 33 & \\
\hline & R3 & ND & $\mathrm{ND}$ & ND & ND & \\
\hline & R4 & 0.19 & 0.32 & $0-0.81$ & 33 & \\
\hline \multirow{4}{*}{ Endosulfanes I y II } & $\mathrm{R} 1$ & 0.62 & 1.3 & $0-2.71$ & 33 & \multirow{4}{*}{10} \\
\hline & R2 & ND & ND & ND & ND & \\
\hline & R3 & ND & ND & ND & ND & \\
\hline & R4 & 5.2 & 12.8 & $0-31.4$ & 33 & \\
\hline \multirow{4}{*}{ DDT } & $\mathrm{R} 1$ & 0.15 & 0.40 & $0-0.90$ & 17 & \multirow{4}{*}{10} \\
\hline & R2 & ND & $N D$ & ND & ND & \\
\hline & R3 & 0.99 & 1.40 & $0-3.31$ & 33 & \\
\hline & R4 & 0.25 & 0.62 & $0-1.53$ & 17 & \\
\hline \multirow{4}{*}{ Endosulfato } & $\mathrm{R} 1$ & ND & ND & ND & ND & \multirow{4}{*}{$\mathrm{NE}$} \\
\hline & R2 & 0.13 & 0.3 & $0-0.8$ & 17 & \\
\hline & R3 & 0.9 & 2.4 & $0-5.9$ & 17 & \\
\hline & R4 & ND & ND & $\mathrm{ND}$ & ND & \\
\hline
\end{tabular}

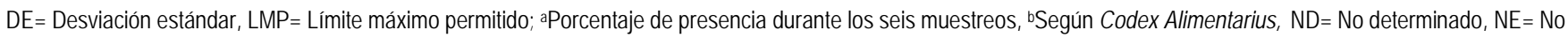
establecido.

considerable (1,992 y 6, 214 casos por año, respectivamente). Aunque no se encontraron datos exactos de los plaguicidas utilizados para el control de vectores de estas enfermedades, considerando que los vientos en esta zona de estudio corren de norte a sur y viceversa, es probable los PO se hayan transportado de Guatemala a la zona de estudio(20).
En relación a las muestras de forraje, los valores más altos fueron alfa+beta-HCH (76.3 $\pm 140 \mathrm{ng} \cdot \mathrm{g}^{-1}$ en R2), lindano (43 \pm 105 ng. g $^{-1}$ en R2), heptacloro + epóxido de heptacloro (40 \pm 76 ng.g ${ }^{-1}$ en R3) y endosulfato (37.5 $\pm 91 \mathrm{ng} \cdot \mathrm{g}^{-1}$ en R3). Los compuestos presentes con mayor frecuencia durante los seis muestreos en las unidades de 
producción fueron alfa + beta- $\mathrm{HCH}$ en $\mathrm{R} 1, \mathrm{y}$ heptacloro + epóxido de heptacloro en R3 con $66 \%$ cada uno (Cuadro 4).

Aunque en México se encuentra prohibido el uso y comercialización de lindano, y en la zona de estudio por su sistema de producción se ha dejado de usar, los resultados obtenidos muestran su probable uso en zonas aledañas. Al realizar la toma de muestras en R1 se observó una bodega con plaguicidas; los productores comentaron que ellos no los usan pero sí los venden a los productores convencionales. Por otro lado, los productores de R2 colindan al norte con productores convencionales quienes aún utilizan lindano como garrapaticida. Lo

Cuadro 3. Cconcentración promedio de algunos plaguicidas organoclorados en muestras de leche orgánica

\begin{tabular}{|c|c|c|c|c|}
\hline \multirow[b]{2}{*}{ Estudios } & \multicolumn{4}{|c|}{ Plaguicidas organoclorados } \\
\hline & $\begin{array}{l}\text { Lindano } \\
\left(\text { ng. }^{-1}\right)\end{array}$ & $\begin{array}{l}\text { Heptacloro + epóxido de } \\
\text { heptacloro }\left(\mathrm{ng} \cdot \mathrm{g}^{-1}\right)\end{array}$ & $\begin{array}{c}\text { Endosulfán I y II } \\
(\text { ng.g.-1) }\end{array}$ & $\begin{array}{l}\text { Aldrín + dieldrín } \\
(\text { ng.g-1 })\end{array}$ \\
\hline Kampire et al., 2012 (Uganda) ${ }^{(5)}$ & 0.026 & 0.002 & 0.002 & $<\mathrm{LD}$ \\
\hline Díaz et al., 2012 (Colombia) (6) & $\mathrm{Nd}^{*}$ & 61.7 & $\mathrm{Nd}$ & 80.1 \\
\hline Luzardo et al., 2012 (Islas Canarias) (7) & 0.08 & 1.14 & 0.0 & 0.66 \\
\hline $\begin{array}{l}\text { Gutiérrez et al., } 2012 \text { (México) (18) } \\
\text { Este estudio (México) }\end{array}$ & $\begin{array}{l}0.34 \\
9.5^{\star *}\end{array}$ & $\begin{array}{l}0.67 \\
24.1\end{array}$ & $\begin{array}{l}1.14 \\
13.2\end{array}$ & $\begin{array}{c}0.77 \\
5.1\end{array}$ \\
\hline
\end{tabular}

$<\mathrm{LD}=$ menor al límite de detección; * no determinado; ${ }^{\star \star}$ concentración promedio de las cuatro unidades.

Cuadro 4. Presencia de plaguicidas organoclorados en muestras de forraje de las unidades de producción analizadas

\begin{tabular}{|c|c|c|c|c|c|}
\hline Compuestos & Unidad & $\begin{array}{c}\text { Promedio } \\
\text { (ng. } .^{-1} \text { base grasa) }\end{array}$ & $\mathrm{DE}$ & $\begin{array}{c}\text { Intervalo } \\
\left.\text { (ng. } g^{-1}\right)\end{array}$ & $\begin{array}{c}\text { Frecuencia } \\
(\%)^{\star}\end{array}$ \\
\hline alfa + beta-HCH & $\begin{array}{l}\text { R1 } \\
\text { R2 } \\
\text { R3 }\end{array}$ & $\begin{array}{l}13.1 \\
76.3 \\
31.6\end{array}$ & $\begin{array}{c}19.7 \\
140 \\
58\end{array}$ & $\begin{array}{l}0-49.6 \\
0-140 \\
0-139\end{array}$ & $\begin{array}{l}66 \\
33 \\
50\end{array}$ \\
\hline Lindano & $\begin{array}{l}\text { R1 } \\
\text { R2 } \\
\text { R3 }\end{array}$ & $\begin{array}{l}0.13 \\
43 \\
9.3 \\
\end{array}$ & $\begin{array}{c}0.23 \\
105 \\
22 \\
\end{array}$ & $\begin{array}{c}0-0.6 \\
0-259.4 \\
0-55\end{array}$ & $\begin{array}{l}33 \\
33 \\
33 \\
\end{array}$ \\
\hline $\begin{array}{l}\text { Heptacloro + epóxido de } \\
\text { heptacloro } \\
\text { Aldrin + dieldrín }\end{array}$ & $\begin{array}{l}\text { R1 } \\
\text { R2 } \\
\text { R3 } \\
\text { R1 } \\
\text { R2 } \\
\text { R3 }\end{array}$ & $\begin{array}{c}15 \\
13.4 \\
40 \\
0.5 \\
4.5 \\
4.0\end{array}$ & $\begin{array}{l}37 \\
21 \\
56 \\
1.3 \\
11 \\
9.0\end{array}$ & $\begin{array}{c}0-91.5 \\
0-31 \\
0-143 \\
0-2.3 \\
0-25 \\
0-22\end{array}$ & $\begin{array}{l}33 \\
50 \\
50 \\
33 \\
33 \\
33\end{array}$ \\
\hline Endosulfán I y II & $\begin{array}{l}\text { R1 } \\
\text { R2 } \\
\text { R3 }\end{array}$ & $\begin{array}{l}5.3 \\
5.0 \\
7.7\end{array}$ & $\begin{array}{l}6.6 \\
7.5 \\
15\end{array}$ & $\begin{array}{c}0-14.1 \\
0-20 \\
0-33\end{array}$ & $\begin{array}{l}50 \\
66 \\
50\end{array}$ \\
\hline DDT & $\begin{array}{l}\text { R1 } \\
\text { R2 } \\
\text { R3 }\end{array}$ & $\begin{array}{l}\mathrm{ND} \\
\mathrm{ND} \\
9.9\end{array}$ & $\begin{array}{l}N D \\
N D \\
21\end{array}$ & $\begin{array}{c}N D \\
N D \\
0-52\end{array}$ & $\begin{array}{l}N D \\
N D \\
50\end{array}$ \\
\hline Endosulfato & $\begin{array}{l}\text { R1 } \\
\text { R2 } \\
\text { R3 }\end{array}$ & $\begin{array}{c}N D \\
9.3 \\
37.4\end{array}$ & $\begin{array}{l}\text { ND } \\
22 \\
91\end{array}$ & $\begin{array}{c}N D \\
0-55 \\
0-224\end{array}$ & $\begin{array}{l}\text { ND } \\
17 \\
17\end{array}$ \\
\hline
\end{tabular}

$\mathrm{DE}=$ desviación estándar, ${ }^{*}$ porcentaje de presencia durante los seis muestreos; ND= no determinado. 
anterior podría justificar, en parte, los valores encontrados en esta unidad de producción ${ }^{(21)}$. Se establece que el estado de Chiapas se encuentra en los primeros lugares en el consumo de plaguicidas y fertilizantes para la producción agrícola (café y plátano, principalmente) y dadas las características ambientales de temperatura y humedad, es altamente probable la movilización de los contaminantes orgánicos que tienden a volatilizarse y depositarse en otros sitios lejanos a la fuente de origen.
A fin de entender mejor el comportamiento de los PO en épocas de seca y de lluvia se realizó un análisis por época del año en las muestras de forraje. En época de seca, los niveles más altos fueron 112 ng. $\mathrm{g}^{-1}$ de alfa-HCH, 86.4 ng. . $^{-1}$ de gama-HCH en R2, $75 \mathrm{ng} \cdot \mathrm{g}^{-1}$ de endosulfato en R3 y $30.8 \mathrm{ng} \cdot \mathrm{g}^{-1}$ de heptacloro en R1 (Figura 1). En época de lluvia los niveles más altos fueron alfa-HCH ( $20 \mathrm{ng} \cdot \mathrm{g}^{-1} \mathrm{en} \mathrm{R1}$, $40 \mathrm{ng} \cdot \mathrm{g}^{-1}$ en R2 y $45 \mathrm{ng} \cdot \mathrm{g}^{-1}$ en R3), heptacloro (41 ng. $\mathrm{g}^{-1}$ en R3) y aldrín (28 $\mathrm{ng} \cdot \mathrm{g}^{-1}$ en R3) (Figura 2). En la zona sur de la República Mexicana, se han reportado concentraciones importantes de

Figura 1. Concentración de plaguicidas organoclorados en muestras de forraje en época de seca

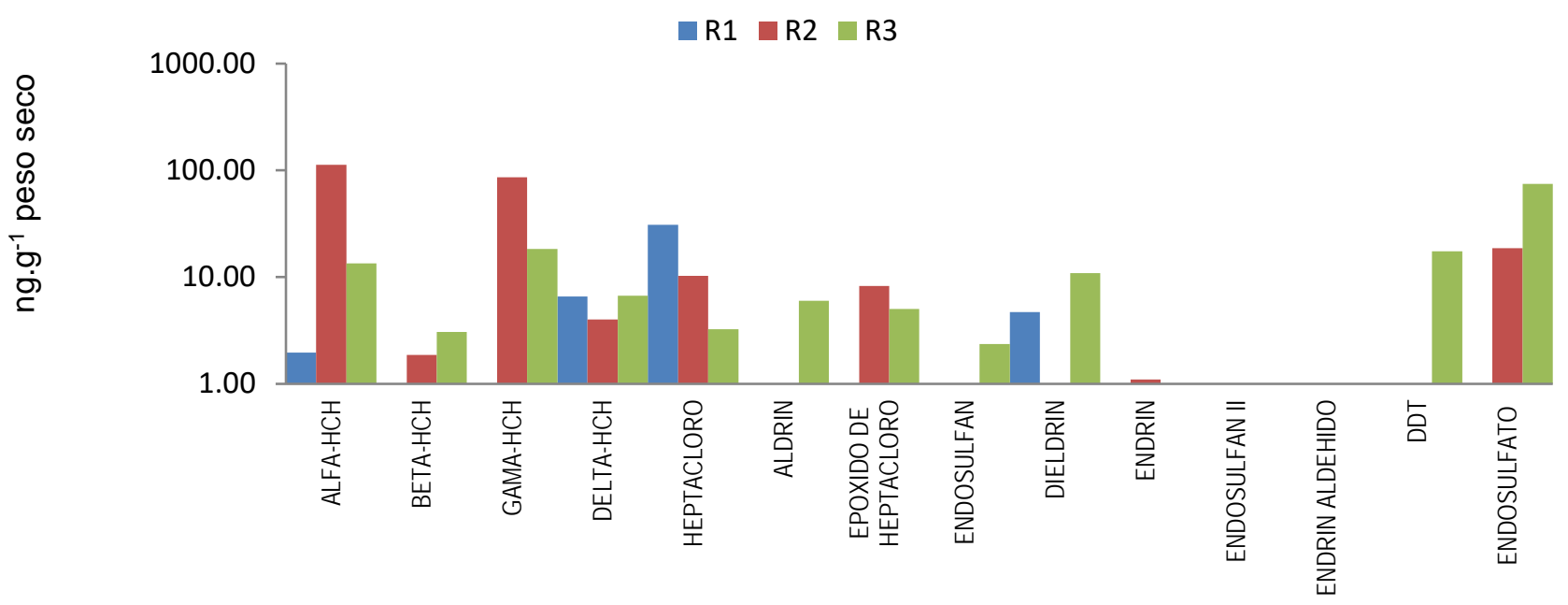

Figura 2. Niveles de plaguicidas organoclorados en muestras de forraje en época de lluvia

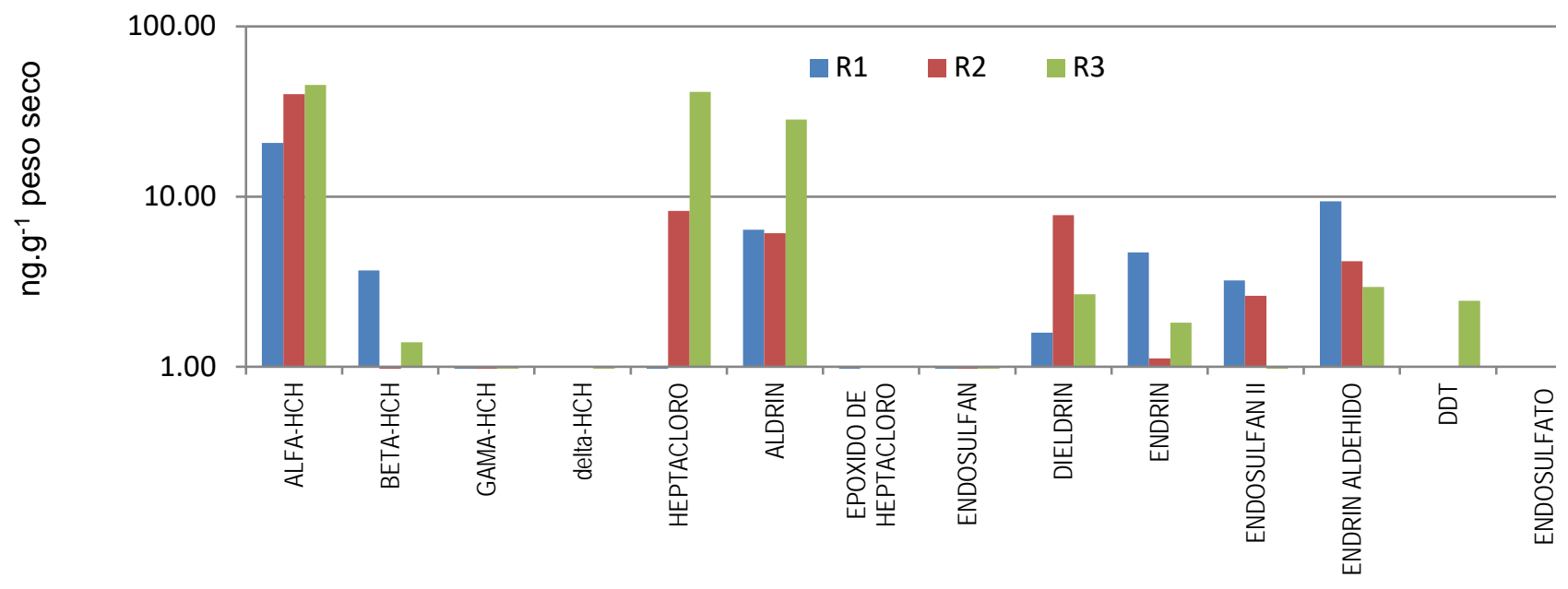


endosulfán en el aire en zonas templadas y tropicales ${ }^{(22,23,24)}$. Por lo que, en las zonas de producción orgánica, los contaminantes pueden entrar al sistema (forraje) a través de la deposición atmosférica, sobre todo compuestos volátiles y semivolátiles. Los factores ambientales juegan un papel importante, ya que se ha descrito que las bajas temperaturas y la lluvia causan un efecto de dilución de contaminantes en la vegetación. Por otro lado, la presencia de plagas y enfermedades favorece la utilización de estos en los cultivos y el ganado $3,25,26)$.

Al no establecerse aún los límites máximos permisibles de PO en forraje, es posible que el problema de contaminación sea variable y se intensifique cuando existen fumigaciones en zonas aledañas a la zona de estudio(3,23). Se han realizado estudios para medir los niveles de PO en hojas de árboles y legumbres, encontrando niveles de alfa$\mathrm{HCH} 22.5 \mathrm{ng} \cdot \mathrm{g}^{-1}$, lindano $10.3 \mathrm{ng} . \mathrm{g}^{-1}$, endosulfán 4.4 ng..$^{-1(27)}$ en hojas de palmera y mango. Sin embargo, no se encontraron referencias sobre la presencia de PO en forraje para ganado lechero.

De forma paralela al presente estudio, se analizaron muestras de agua de los abrevaderos de las vacas, así como del suelo. Los plaguicidas que se encontraron en mayor concentración fueron alfa + beta-HCH con 0.09 ng. $\mathrm{ml}^{-1}$ en las muestras de agua y $0.99 \mathrm{ng}^{-1} \mathrm{~g}^{-1}$ en suelo. Dichos datos se encuentran propuestos en otra publicación. Existen pocos estudios que analicen la presencia de contaminantes orgánicos persistentes, tales como los plaguicidas organoclorados en diversos compartimentos ambientales (suelo, agua, forraje, leche y derivados) y que pongan en evidencia la magnificación de estos. Los pocos existentes se han realizado en zonas costeras con énfasis a la producción pesquera - alteración de ciclos de vida de especies comerciales ${ }^{(28)}$. Sin embargo, para las zonas de producción orgánica no existen estudios detallados y menos aún en hatos lecheros orgánicos. En el presente estudio se observaron concentraciones más altas en las muestras de leche que en las de forraje (Figura 3), probablemente por la acumulación constante de dichos compuestos a través de la inhalación de vapores por el rocío de estos compuestos en un lugar cercano (plantaciones) y la ingesta de alimento contaminado (forraje). Por lo que es necesario monitorear debidamente las actividades cercanas a la zona de producción lechera para reducir las concentraciones de plaguicidas en el ambiente, y con ello en los productos de consumo para los organismos.

Figura 3. Niveles de plaguicidas en muestras de forraje y leche orgánica en las unidades de producción

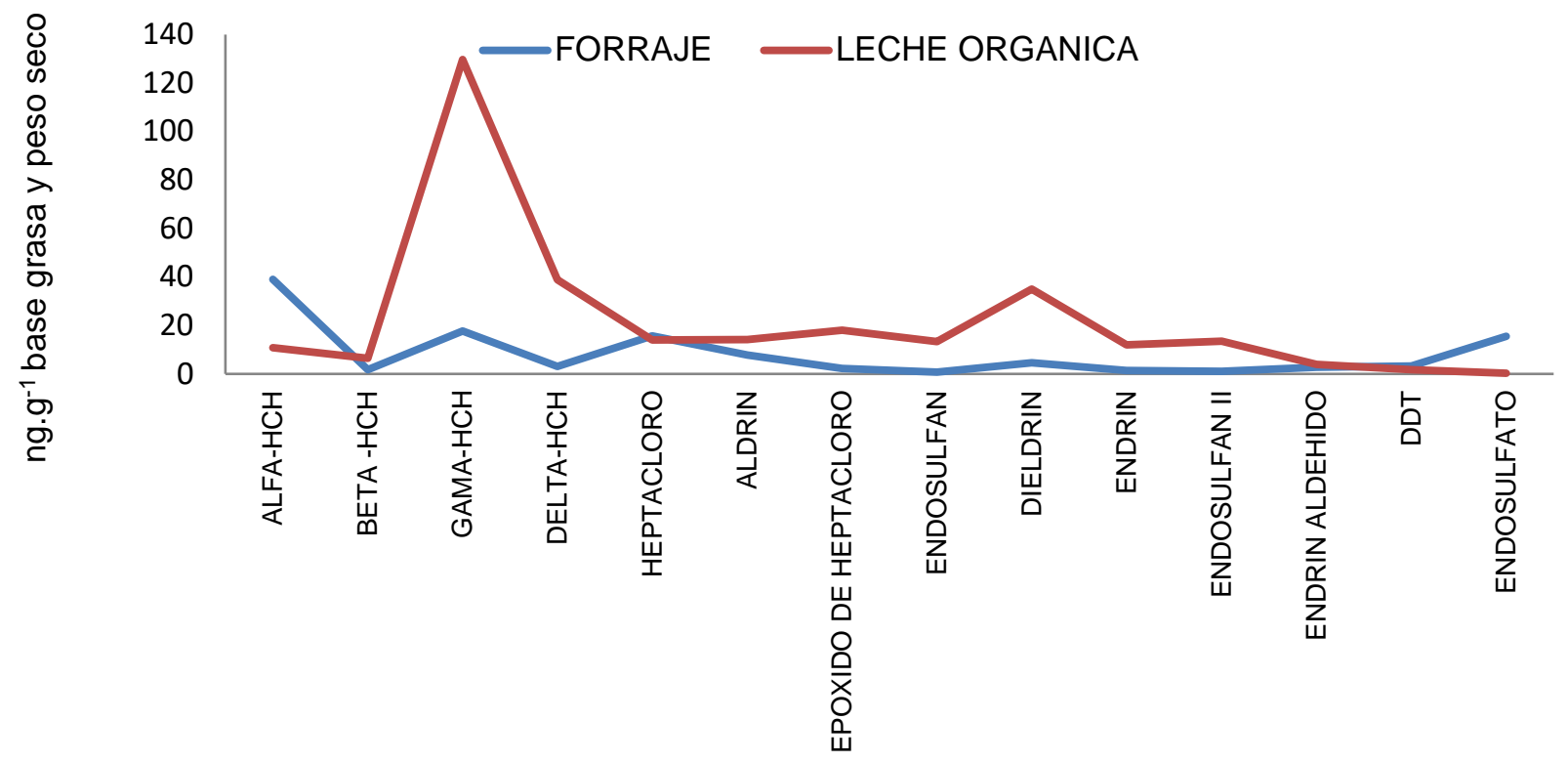




\section{CONCLUSIONES E IMPLICACIONES}

Se lograron detectar niveles de PO en las muestras de forraje y en mayor concentración en muestras de leche orgánica, reflejando una probable biomagnificación de estos compuestos. Aunque en México existe reglamentación y convenios a nivel internacional para el uso adecuado de PO para proteger a las poblaciones y los ecosistemas de sus efectos, no hay un límite máximo establecido para forraje. Tomando en cuenta el aumento de la producción de alimentos orgánicos, es importante continuar con el estudio de contaminantes persistentes en muestras ambientales (suelo, agua, forraje) en unidades de producción orgánica, con el fin de determinar su comportamiento a lo largo del tiempo, y así disminuir en lo posible la exposición a estos compuestos por el consumo de alimentos.

\section{AGRADECIMIENTOS}

Consejo de Ciencia y Tecnología (CONACyT), por el financiamiento otorgado para la realización del presente estudio. Laboratorio de Análisis Instrumental de la Universidad Autónoma Metropolitana por las facilidades en la realización del análisis de plaguicidas. Facultad de Medicina Veterinaria y Zootecnia de la Universidad Nacional Autónoma de Chiapas, por las facilidades prestadas durante el trabajo de campo. Dr. Rutilio Ortiz Salinas, profesor del Dpto. de Producción Agrícola y Animal de la Universidad Autónoma Metropolitana unidad Xochimilco por la revisión crítica del trabajo.

\section{LITERATURA CITADA}

1. Sojinu S; Sonibare O, Ekundayo O, Zeng E. Assessment of organochlorine pesticides residues in higher plants from oil exploration areas of Niger Delta, Nigeria. Sci Total Environ 2012;433:169-177.

2. Mahugija J, Kishimba M. Organochlorine pesticides and metabolites in young leaves of Mangifera indica from sities near a point source in Coast region, Tanzania. Chemosphere 2007;68:832-837.

3. Yang $X$, Jiang $X, Y u$ G, Yao F, Bian $Y$, Wang F. Leaf-air transfer of organochlorine pesticides from three selected vegetables. Environ Poll 2007; 148:555-561.

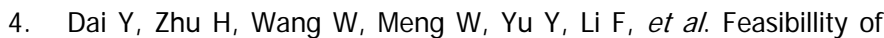
popular foliages as bio-monitors for organochlorine pesticides in air Chinese. Sci Bull 2009;54(9):1515-1521.
5. Kampire E, Kiremire B, Nyanzi S, Kishimba M. Organochlorine pesticides in fresh and pasteurized cow's milk from Kampala markets. Chemosphere 2011;84:923-927.

6. Díaz PB, Lans CE, Barrera VJL. Residuos de insecticidas organoclorados presentes en leche cruda comercializada en el departamento de Córdoba, Colombia. Acta Agron 2012;61(1):1015.

7. Luzardo O, Almeida M, Enríquez LA, Zumbado M, Álvarez E, Boada LD. Polychlorobiphenyls and organochlorine pesticides in conventional and organic brands of milk: Occurrence and dietary intake in the population of the Canary Islands (Spain). Chemosphere 2012;88:307-315.

8. Ruíz J L. Producción de leche orgánica en el municipio de Tecpatán Chiapas. 2nd ed. Chiapas, México: Talleres Gráficos UNACH; 2008.

9. Gómez MA, Schwentesius R, Ortigoza J, Gómez L. Situación y desafíos del sector orgánico de México. Rev Mex Cienc Agríc 2010;1(4):593-603.

10. Ruíz JL, Nahed J, Gutiérrez R, Velasco ME, Yamasaki A. La producción de leche orgánica en Chiapas. 2a ed. Chiapas, México: Talleres Gráficos UNACH; 2011.

11. IFOAM (International Federation of Organic Agriculture Movements) Normas de IFOAM para la producción y el procesamiento orgánicos. http://.www.ifoam.org. Consultado 23 Sep, 2013.

12. INEGI (Instituto Nacional de Estadística y Geografía) (2010). Prontuario de información geográfica municipal de los Estados Unidos Mexicanos http://www.inegi.org.mx/sistemas/mexicocifras/ datos-geograficos/07/07092.pdf. Consultado 15 Oct, 2011.

13. Norma Mexicana NMX-Y-111-SCFI-2010. Alimentos para animales. Muestreo de alimentos balanceados e ingredientes mayores. Diario Oficial de la Federación. 14/03/11.

14. Consejo para el Fomento de la Calidad de la Leche y sus derivados (COFOCALEC) NMX-F-718-COFOCALEC-2006. Sistema Producto Leche-Alimentos-Lácteos Guía para el muestreo de leche y productos lácteos. http://cofocalec.org.mx/internaindustrias. php? tipo=0\&id=11. Consultado 16 J un, 2013.

15. Frank R, Smith EH, Holdrinet $M$, McWade JW. Organochlorine Insecticides and industrial Popullants in the milk supply of the Sourtherrn Region of Ontario. Can J Milk Food Technol $1975 ; 38(2): 65-72$.

16. USEPA (United States Environmental Protection Agency). Determination of DDT's, PCB`s and other hydrocarbons in marine sediments by gas liquid chromatography. Reference methods for marine pollution studies. 1981.

17. Hernández M, Vidal J, Marrugo J. Plaguicidas organoclorados en leche de bovinos suplementados con residuos de algodón en San Pedro, Colombia. Rev Salud Púb 2010;12(8):982-989.

18. Gutiérrez R, Ruíz JL, Ortíz R, Vega S, Schettino B, Yamasaki A, Ramírez ML. Organochlorine pesticides residues in bovine milk from organics farms in Chiapas, Mexico. Bull Environ Contam Tox 2012; 89:882-887.

19. Ruíz RE, Ruíz JA, Gúzman S, Pérez EJ. Manejo y control de plagas del cultivo de tomate en Cintalapa, Chiapas, México. Revista Int Contam Ambient 2011;27(2):129-137. 
20. Ministerio de Salud Pública Guatemala. 2015 www.mspas.gob.gt consultado 11 J un, 2015.

21. Ortiz I, Ávila A, Torres L. Plaguicidas en México: usos, riesgos y marco regulatorio. Rev Latinoam Biotecnol Amb Algal 2013;4(1):26-46.

22. Barakat A, Mostafa A, Wade T, Sweet S, El-Sayed N. Distribution and ecological risk of organochlorine pesticides and polychlorinated biphenyls in sediments from the Mediterranean coastal environment of Egypt. Chemosphere 2013;93:545-554.

23. Alegria HA, Bidleman TF, Salvador M. Organochlorine pesticides in the ambient air of Chiapas, Mexico. Environ Poll 2006; 140:483-491.

24. Becker I, Scheringer M, Schenker U, Hungerbühler K. Assessment of the environmental persistence and long-range transport of endosulfan. Environ Poll 2011;159:1737-1743.
25. Costera A, Feidt $C$, Marchand P, Le B,Rychen G. PCDD/F and PCB transfer to milk in goats exposed to a long term intake of contaminated hay. Chemosphere 2006; 64:650-657.

26. Mansour AS, Belal HM, Abou-Arab AK, Gad FM. Monitoring of pesticides and heavy metals in cucumber fruits produced from different farming systems. Chemosphere 2009; 75:601-609.

27. Sojinu S, Sonobare O, Ekundayo O, Zeng E. Assessment of organochlorine pesticides residues in higher plants from oil exploration areas of Niger Delta, Nigeria. Sci Total Environ 2012; 433:169-177.

28. Espinosa G, Hizaliturri C, González D, Mejía J, Nava AD, Cuevas MA, Cilia G. Contaminantes orgánicos persistentes en la cuenca baja del río Coatzacoalcos, Veracruz. En: Botello AV, et a/ editores. Golfo de México. Contaminación e impacto ambiental: diagnóstico y tendencias. UAC, UNAM-ICMYL, CINVESTAV- Unidad Mérida; 2013:309-322. 